\title{
Jejunal diverticulosis: Report of a case and review of the literature
}

\author{
WILLIE JAMES CHESTER, DO \\ JOHN E THORNBURG, DO, PHD
}

\begin{abstract}
A report of a case and review of the literature of jejunal diverticulum are presented. A 45-year-old woman presented with crampy, generalized abdominal pain which had been present intermittently for five years. A complete endoscopic examination of the gastrointestinal tract was precipitated by an acute decrease in hemoglobin level. The diagnosis was made by detailed small bowel study. In a review of the literature, clinical features, difficulties in diagnosis, and complications are emphasized.
\end{abstract}

The Latin origin of the word diverticulum designates such structures, when found in the intestine, as byways or wayside prolongations of the lumen. Diverticula of the jejunum traditionally have been accorded benign status. However, the increasing number of reports that associate significant abnormalities to jejunal diverticula suggests that they should be regarded more seriously.

Patients with jejunal diverticulosis may be labeled psychoneurotic because most investigative studies fail to demonstrate organic disease. ${ }^{1,2}$ A typical patient complains of intermittent, frequently cramping abdominal pain, which usually is brought on or aggravated by eating. The pain is associated with nausea, vomiting, belching, flatulence, diarrhea, or constipation. Anemia and malnutrition may develop. Frequently, because of chronic gastrointestinal symptoms, the patient has had repeated roentgenographic examinations and has been seen by several physicians over a period of years. Although usually asymptomatic, diverticula can be associated with massive gastrointestinal bleeding, perforation, obstruction, abscess formation, and malabsorption. The case described below illustrates some of the difficulties in the diagnosis of jejunal diverticulosis. The literature describing the features of the condition is then summarized.

\section{Report of case}

A 45-year-old white woman was first seen in the clinic on January 25, 1986, because of periodic stomach ache of five years' duration. Her abdominal discomfort was described as crampy and generalized, but worse in the right upper quadrant and epigastric regions. Onset was approximately 60 to 90 minutes after eating. Bloating, marked borborygmi, and flatus were always associated with pain. The patient stated that she had noted foulsmelling watery stools on and off for the past six months; dark red blood mixed with the stool had been noted for three months. There was no history of weight loss. The patient emphasized the intermittent nature of the pain, stating that she could sometimes eat without difficulty, while there were other times when eating resulted in severe cramping or vomiting.

She had a past medical history of appendicitis, bronchitis, and vaginitis, and a surgical history of appendectomy, tonsillectomy, and right ovarian cystectomy. The family history was positive for peptic ulcer disease, diabetes mellitus, rheumatic fever, and cancer of the colon, uterus, and vulva. She had no known allergies and did not use tobacco or ethanol. She had taken Bentyl and Mylanta in the past, which had resulted in minimal to moderate relief of her abdominal symptoms.

The physical examination was essentially negative, with the exception of mild epigastric discomfort on palpation. Ultrasonography of the gallbladder was negative for gallstones or signs of infection. Hemoglobin and hematocrit values were $11.4 \mathrm{~g} / \mathrm{dL}$ and $32.2 \%$, respectively; stool was negative for occult blood. The patient was diagnosed as having irritable bowel syndrome with possible peptic ulcer disease, treated with ranitidine, and asked to return in one week. However, she had a synco- 
pal episode within the following 48 hours and was hospitalized. Her hemoglobin level had decreased from 11.4 to $8.4 \mathrm{~g} / \mathrm{dL}$ within the two-day period,

The patient was given packed red blood cells replacement, and her hemoglobin level subsequently stabilized and slowly increased. Upper gastroinestinal endoscopy, sigmoidoscopy, and colonoscopy failed to demonstrate any active bleeding sites. A detailed small-bowel study demonstrated two small hernial diverticula in the proximal jejunum. She was discharged on the eighth hospital day in stable condition and has been followed as an outpatient on a weekly basis with no evidence of recurrent bleeding or anemia, or complaints of abdominal pain or discomfort. She was given a two-week course of oral antibiotic treatment, (metronidazole, $500 \mathrm{mg}$., 3 times a day). The patient declined further antibiotic treatment.

\section{Discussion}

\section{Pathologic anatomy and physiology}

Hernial diverticula of the small intestine appear as rounded or elliptic, single or multiloculated outpouchings of the intestinal wall. The sac wall is thin and is composed largely of mucosal and adventitious submucosal fibrous tissue. It lacks the well-developed smooth muscle layers that are characteristic of the normal, adjacent small intestine. Hernial diverticula is the type that often becomes pathologic. The terms acquired, pseudo, false, or pulsion diverticula are used interchangeably to denote these structures, in contradistinction to the other form of intestinal diverticulum, which is known as congenital or true diverticulum. ${ }^{2,3}$

The pathogenesis of these false diverticula is unclear, but they may result from an abnormal increase in intraluminal pressure in the small intestine ${ }^{3,4}$ Relative weakness of the bowel at the sites of neurovascular entry plus high intraluminal pressure allow protrusion of the mucosa and submucosa. $^{2}$

Devoid of muscle and composed only of mucosa and submucosa, the atonic diverticula empty poorly. The absence of normal peristalsis may be important in the etiology of malabsorption, which is secondary to bacterial overgrowth.

\section{Incidence, age and sex distribution}

Jejunal diverticulosis is an infrequent radiologic or surgical finding. The reported frequency at autopsy approximates $0.2 \% .{ }^{4}$ Because these diverticula are acquired structures, they are seen increasingly with aging; the highest incidence is in the sixth and seventh decades of life.$^{5,6}$ Although the disorder is rarely seen before the age of 40 , one case in a 10-year-old child has been reported. ${ }^{6}$ In a total of 48 cases studied by Taylor ${ }^{5}$ and Nobles, ${ }^{6} 31$ patients were male and 17 were female (1.8:1 male predominance).

\section{Clinical features and symptoms}

It is estimated ${ }^{2,5}$ that only a small percentage of patients have symptoms that are clearly attributable to small-bowel diverticula. Because gastrointestinal workup may not demonstrate an organic problem, the patient may be thought to be neurotic. ${ }^{1,2}$ Typically, the patient reports periodic abdominal pain (usually cramping), which follows or is made worse by ingestion. Nausea and vomiting, flatulence and belching, and diarrhea or constipation often accompany the pain.

As in the present case, patients frequently are diagnosed as having irritable bowel syndrome and are treated accordingly. However, over a period of years, complications may occur.

\section{Complications}

Although rare, complications of the condition may be the presenting features of jejunal diverticula. Acute complications include massive hemorrhage, perforation, and obstruction, while anemia, malabsorption, and hypoproteinemia are chronic complications. $^{\text {? }}$

Hemorrhage from jejunal diverticula occurs infrequently. ${ }^{4}$ The patient may present with vomiting of bright red blood or coffee grounds material; however, melena is the most common presenting complaint. ${ }^{5}$ In most cases, the bleeding is massive (decrease in hemoglobin level of 3-4 g/dL, but stops spontaneously). Following an initial hemorrhage, the probability of further bleeding increases..$^{1,4}$

Of the 48 patients in the studies of Taylor and Nobles, nine were in their $40 \mathrm{~s}$. Of these, five had massive hemorrhage, one had nonmassive melena, and one vomited blood. There has been no report of hemorrhage in patients under the age of 40 . Twenty-seven of the 48 total cases occurred after the age of 60 . With an increasing population of older persons, jejunal diverticula may prove to be of greater importance as a cause of gastrointestinal bleeding.

Perforation has been reported in less than 50 cases of jejunal diverticula. Perforation can present as spontaneous asymptomatic pneumoperitonitis or as diverticulitis with localized or generalized peritonitis. ${ }^{8}$ Fecal contamination may enter the peritoneal cavity through minute perforations in the wall of the diverticula as a result of hyperactive peristaltic activity.

Obstruction, an uncommon complication, is attributed ${ }^{1}$ to thickened hypertrophied walls of the jejunum associated with a large number of thinwalled diverticula. This results in jejunal dysfunction or dyskinesia, the more severe type of which would present with obstruction. Most often, mild 
partial obstruction is associated with the symptoms of pain, distention, flatulence, and borborygmus, which become more severe as the diverticula enlarges. ${ }^{4}$ Hypoproteinemia is a frequent manifestation of the blind loop syndrome and may be severe enough to cause edema. Jejunal diverticulosis is one of many lesions that may cause the blind loop syndrome-vitamin $\mathrm{B}_{12}$ malabsorption, megaloblastic anemia, and steatorrhea. This triad is rare, however, with only 24 cases having been recorded in the literature. ${ }^{9}$

Bacterial overgrowth is the common factor in all malabsorption problems stemming from intestinal stasis. ${ }^{10}$ Multiple factors may be responsible for bacterial overgrowth: the emptying ability of smallbowel diverticula, which are usually deficient of small-bowel muscle layers; the absence of normal peristalsis; and the less acid $\mathrm{pH}$ of the proximal small bowel. Bacterial utilization of vitamin $\mathrm{B}_{12}$ in a competitive manner, as well as bacterial product interference with its absorption, have been postulated as mechanisms by which vitamin $\mathrm{B}_{12}$ deficiency occurs. ${ }^{9}$

\section{Diagnosis}

The diagnosis of jejunal diverticula can be difficult. Physical examination usually is noncontributory. The syndrome of vague abdominal pain, anemia of obscure origin, and slightly dilated loops of jejunum points to jejunal diverticulosis. ${ }^{4}$ The diagnosis usually is made by abdominal roentgenograms demonstrating air-fluid levels or barium in the diverticula. The frequency of diagnosis depends on the skill and awareness of the radiologist. Diagnosis may be confirmed at surgery, but diverticula hidden by loops of adherent jejunum or buried in the mesentery may be overlooked. ${ }^{2}$ Of course, if the patient presents with active gastrointestinal bleeding, angiography will identify the site if blood loss is $\geqslant 5 \mathrm{~mL} / \mathrm{min}$.

\section{Treatment}

Medical therapy for symptomatic jejunal diverticulosis involves management of complications such as diverticulitis, small-bowel bacterial overgrowth, and vitamin $\mathrm{B}_{12}$ deficiency. Broad-spectrum antibiotics are used in episodic courses of one to two weeks per month to suppress bacterial overgrowth. Symptoms of diarrhea, bloating, and significant weight loss often will improve with this therapy. ${ }^{7}$ Parenteral $\mathrm{B}_{12}$ can be given to replete losses of this vitamin.

There is little information on the use of motilitypromoting agents, such as metoclopramide, in the treatment of pseudo-obstruction associated with jejunal diverticulosis. However, an empiric trial in an attempt to enhance upper gastrointestinal motility may be considered.

Surgical resection generally is reserved for patients with serious complications, such as hemorrhage, perforation, and complete small bowel obstruction.

\section{Summary}

The jejunum is one of the least likely sites of the gastrointestinal tract to be affected by diverticulosis. The condition is almost twice as common in men, and it occurs most frequently in the sixth and seventh decades of life. Abdominal complaints are present in only $10 \%$ to $20 \%$ of affected individuals; in most cases these consist of postprandial abdominal pain, distention, flatulence, nausea, and vomiting. Rarely are acute symptoms, such as massive gastrointestinal hemorrhage, obstruction, or perforation, the initial presenting complaint.

1. Altemeier WA, Bryant LR, Wulsin, JH: The surgical significance of jejunal diverticulosis. Arch Surg 1963;86:732-744.

2. Christensen N: Jejunal diverticulosis. Am J Surg 1969;118:612-618.

3. Pinto DJ: Jejunal diverticulosis in the presence of small bowel obstruction. Br J Surg 1968;55:264-265.

4. Forse RA, Tabah EJ: Jejunal diverticulosis. Can J Surg 1980;23:291293.

5. Taylor MT: Massive hemorrhage from jejunal diverticulosis. Am $J$ Surg 1969;118:117-120.

6. Nobles ER: Jejunal diverticula. Arch Surg 1971;192:172-174.

7. McGrew W, Patel J, Miller P: Jejunal diverticulosis: Medical and surgical management. South Med J 1985;78:533-535.

8. Herrington JL. Spontaneous asymptomatic pneumoperitoneum. Am $J$ Surg 1967;113:567-570.

9. Knauer CM, Svoboda AC: Malabsorption and jejunal diverticulosis. Am $J$ Med 1968;44:606-610.

10. Williams RA, Davidson DD, Serota A, et al: Surgical problems of diverticula of the small intestine. Surg Gynecol Obstet 1981;152:621626.

From the Department of Family Medicine (Dr Chester) and Department of Pharmacology and Toxicology (Dr Thornburg), Michigan State University-College of Osteopathic Medicine, East Lansing, Mich.

For reprints, address Dr. Thornburg, MSU-COM, Department of Pharmacology and Toxicology, Life Sciences Bldg, East Lan- 


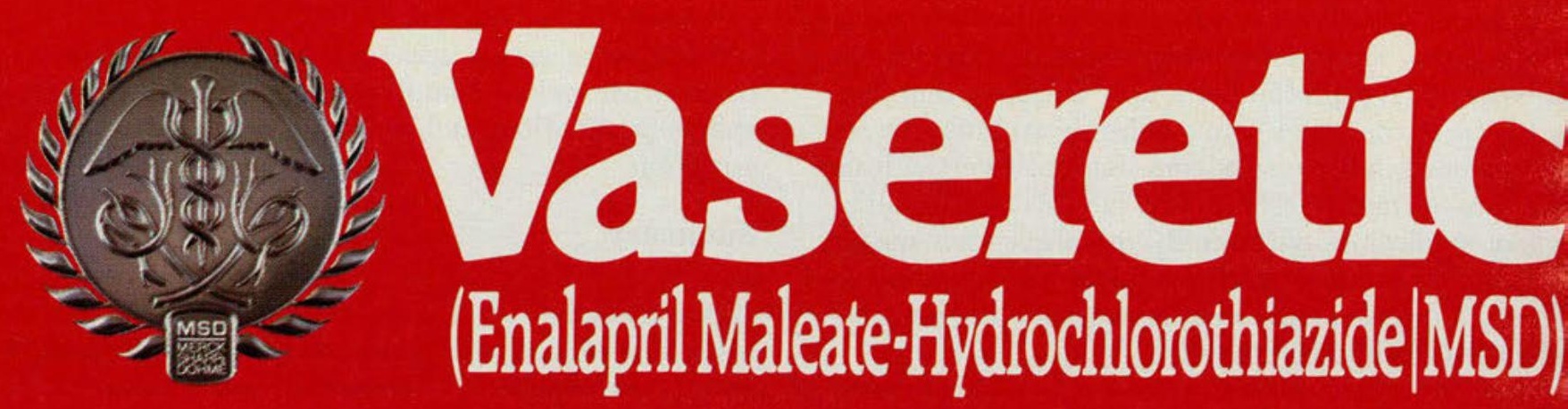

\section{For added hypertension control}

Hydrochlorothiazide augments the antihypertensive effect of enalapril.

Enalapril tends to reverse the potassium loss often associated with hydrochlorothiazide.

Highly effective in adults, regardless of age or race. 


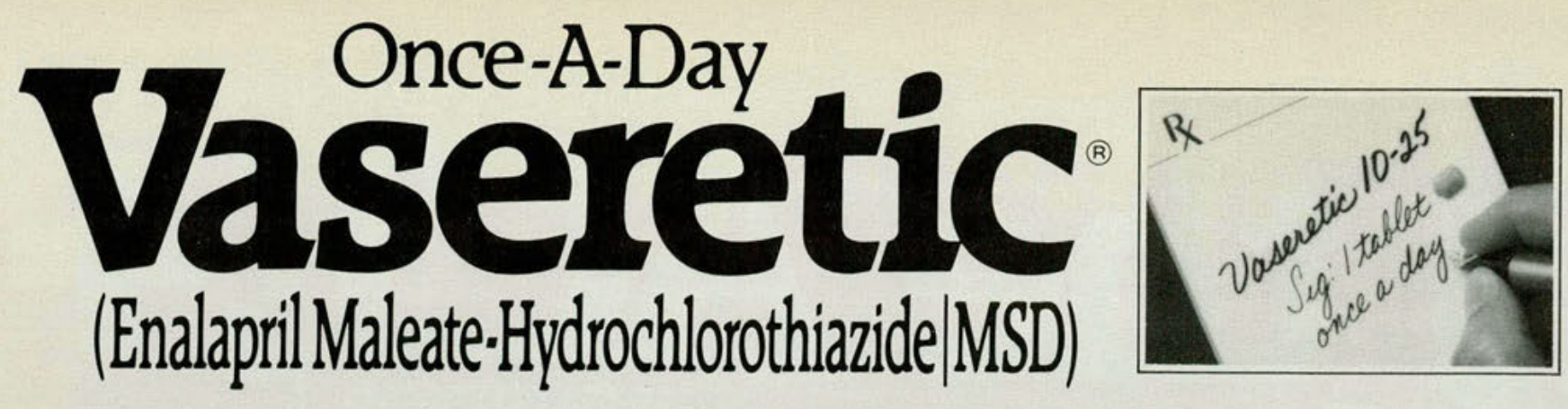

This fixed-dose combination is not indicated for initial therapy. Patients already receiving a diuretic when enalapril is initiated, or given a diuretic initial titration of the individual entities. it is important, if possible, to stop the diuretic for several days betore starting enalapril or. if this is not possi-
ble. begin enalapril at a low initial dose (see DOSAGE AND ADMINISTRAON in complete Prescribing Information). This fixed-dose combination is (t) nents if the litrated doses

VASERETIC \& (Enalapril Maleate-Hydrochlorothiazide. MSO) is contraind Because of the hydrochlorothiazide component. this product is contraindicated in patients with anuria or hypersensitivity to other sulfonamide-derived
drugs WARNINGS

Hypotension:

Excessive hypotension was rarely seen in uncomplicated diuretics or patients on dialysis. Syncope has been reported in $1.3 \%$ o dence of syncope is $0.5 \%$. The overall incidence of syncope may be reduced by proper ttration of the individual components (see PRECAUYTIONS, Drug
Interactions: ADVERSE REACTIONS: and DOSAGE AND ADMINISTRATION in complete Prescribing Information). In patients with severe congestive heart has been observed and may be associated with oliguria and or progressive potential fall in blood pressure in these patients, therapy should be started under very close medical supervision. Such patients should be followed
closely for the first two weeks of treatment and whenever the dose of enalapri

If hypotension occurs, the patient should be placed in a supine position and. tensive response is not a contraindication to further doses. Which usually can

Angioedema: Angioedema of the face, extremities. lips, tongue. glottis. and or larynx has been reported in patients treated with angiotensin-converting-
enzyme inhibitors. including enalapril. In such cases. VASERETC should be disappears. In instances where swelling has been confined to the face and lips

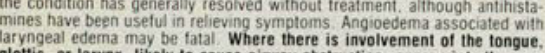
glottis, or larynx, likely to cause airway obstruction, appropriate therapy

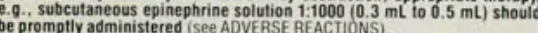
Neutropenia Agranulocytosis: Another angiotes

inhibitor has been shown to cause agranulocylosis and bone marrow depres. renai impairment, especially if they also have a collagen vascular disease
Available data from clinical trials of enalaprilare insulficient to show that enal april does not cause agranulocytosis at similar rates. Foreign marketing expe-
rience has revealed several cases of neutropenia or agranulocytosis in which causal relationship to enaiapril cannot be exciuded Periodic monitoring of
white blood cell counts in patients with collagen vascular disease and renal
disease should be considered Hydrochlorothiazide: Thiazides should be used with caution in severe rena disease. In patients with renal disease, thiazides may precipitate azotemia
Cumulative effects of the orug may develop in patients with imparred renal Thiazides should be used with caution in patients with impaired hepatic function or progressive liver disease. since minor
lyte balance may precipitate hepatic coma.

Sensitivity reactions may occur in patients with or without a history of allergy

The possibility of ex

Lithium generally should not be given with thiazides (see PRECAUTIONS PRECAUTIONS

General: Enalapril Maleate: Impaired Renal Function: As a consequence of inhibiting the renin-angiotensin-aldosterone system, changes in renal func-
tion may be anticipated in susceptible individuals. In patients with severe congestive heart failure whose renal function may depend on the activity of the
renin-angiotensin-aldosterone system, treatment with angiotensin-converting-ennyme inhibitors, including enalapril. may be associated with oliguria
and or progressive azotemia and rarely with acute renal failure and or death In clinical studies in hypertensive patients with unilateral of bilateral renal
artery stenosis. increases in bilood urea nitrogen and serum creatinine were artery stenosis, increases in biood urea nitrogen and serum creatinine were
observed in $20 \%$ of patients . These increases were almost always reversible
upon discontinuation of enalapri and or diuretic therapy in such patients.
renal function should be monitored during the first few weeks of therapy. Some hypertensive patients with no apparent pre-existing renal vascular dis-
ease have developed increases in blood urea and serum creatinine. usually
minor and transient. especially when enalapril has been given concomitantly with a diuretic. This is more likety to occur in patients with pre-existing rena
impairment. Dosage reduction of enalapril and or discontinuation of the
diuretic may be required. Evaluation of the hypertensive patient should always include assessment of renal function.

Hyperkalemia: Elevated serum potassium (greater than $5.7 \mathrm{mEg} / \mathrm{L}$ ) was
observed in approximately $1 \%$ of hypertensive patients in clinical trials treated with enalapril alone in most cases. these were isolated values which resolved
despite continued therapy. although hyperkalemia was a cause of discontinuation of therapy in $0.28 \%$ of hypertensive patients. Hyperkalemia was less tre. hydrochlorothiazide Risk factors for the deveiopment of hyperkalemia may include renal insutficiency. diabetes mellitus. and the concomitant use of

Surgery / Anesthesia: In patients undergoing major surgery or during anesthe-
sia with agents that produce hypotension, enalapril may block angiotensin II lormation secondary to compensatory renin release. If hypotension occurs
and is considered to be due to this mechanism, it can be corrected by volume

Hydrochlorothiazide: Periodic determination of serum electrolytes to detect possible electrolyte imbalance should be performed at appropriate intervals,
All patients receiving thiazide therapy should be observed for clinical signs of
fluid or electrolyte inbalance: namely hyponatremia. hypochloremic alkalofluid or electrolyte imbalance: namely hyponatremia. hypochloremic alkalo-
sis. and hypokalemia. Serum and urine electrolyte determinations are particu-
larly important when the patient is vomiting excessively of receiving parenteral fluids. Warning signs or symptoms of fluid and electrolyte imbalance. irrespective of cause, include dryness of mouth, thirst, weakness. tatigue, hypotension, oliguria, tachycardia, and gastrointestinal disturbances
such as nausea and vomiting sypokalemia may develop. especially with brisk diuresis, when severe cirrth trolyte intake will also contribute to hypokalemia. Hypokalema can sensitize or exaggerate the response of the hearn to the loxic effects of digitalis (e. 9 . aldosterone. concomitant therapy with enalapril attenuates the diuretic-
induced potassium loss isee Orug Interactions. Agents increasing Serum Although any chionde deficit is generally mild and usually does not require specitic treatment except under extraordinary circumstances las in liver dis. ease or renal disease). chlotide replacement may be required in the treatment
of metabolic alkalosis. appropriate therapy is water restriction rather than administration of sat except in rare instances when the hyponatremia is lite-threatening. In actu Hyperuricemia may occur or frank gout may be precipitated in certain patients
receiving thiazide therapy. In diabetic patients. dosage adjustments of insulin or oral hypoglycemic
agents may be required. Ayperglycemia may occur with thiazide diuretics
Thus, latent diabetes mellitus may become manifest during thiazide therapy The antihypertensive effects of the drug may be enhanced in the postsy If progressive renal impairment
discontinuing diuretic therapy.

Thiazides have been shown to increase the urinary excretion of magnesium Thiazides may decrease urinary calcium excretion. Thiazides may cause inte-
mittent and slight elevation of serum calcium in the absence of known diso ders of calcium metabolism. Marked hypercalcemia may be evidence of Information for Patients: Angioedema: Angioedema. including laryngea
edema. may occur especially following the first dose of enalaprit. Patient should be so advised and told to report immediately any signs or symptoms
suggesting angioedema (swelling of tace extremities. eves. lips. tongue and suggesting angioederna (swelling of tace. extremities eyes. lips. tongue and
or difficulty in breathing) and to take no more drug unti they have consulted Hypotension: Patients should be cautioned to report lightheadedness espe.
clally during the first few days of therapy. If actual syncope occurs, patients should be told to discontinue the drug until they have consulted with the pre-
scribing physician. All patients should be cautioned that excessive perspiration and dehydration
may lead to an excessive faili in biood pressure because of reduction in fluid
volume. Other causes of volume deplefion such as vomiting or diarriea may also lead to a fall in blood pressure. patients should be advised to consult with
the physician.
Hyperkalemia: Patients should be told not to use salt substitutes containing Neutropenia: Patients should be told to report promptly any indicat
infection (e. 9. Sore throat, fever) which may be a sign of neutropenia NOTE: As with many other drugs. certain advice to patients being treated with
VASERETIC 8 (Enalapril Maleate-Hydrochlorothiazide. MSO) is warranted fion. It is not a disclosure of all possible adverse or intended effects. Orug Interactions: Enalapril Maleate: Hypotension-Patients on Diuretic was recently instituted may occasionally experience an excessive reduction of hypotensive effects with enalapril can be minimized by either discontinuing
the duretic oo increasing the salt intake prior to initiation of treatment with
enalapril. If it is necessary to continue the diuretic. provide medical superv. Sion for at least one hour after the initial dose (see WARNINGS and DOSAGE
AND ADMINISTRATION). Agents Causing Renin Release. The antihypertensive effect of enalapnil is augmented by antihypertensive agents that cause been used concomitantly with beta-adrenergic-blocking agents, methyldopa. sium: Enalapril attenuates diuretic-induced potassium loss. Potassium-spar suppliements. or potassium-containing salt substitutes may lead to sotasniticant
increases in serum potassium. Therefore, if concomitant use of these agents serum potassium.
Hydrochlorothiazide: When administered concurrently the following drugs Hydrochlorothiazide: When administered concurrently, the following drugs
may interact with thiazide diuretics. Alcohol, barbiturates, or narcoticspotentiation of orthostatic hypotension may occur. Antidiabetic drugs (oral
agents and insulin) - dosage adjustment of the antidiabetic drug may be
required. Other antitypertensive drugs - additive effect or potentiation. Cor

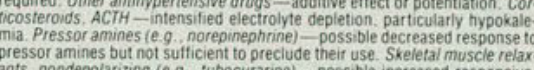
ants. nondepolarizing (e.g. tubocurarine)-possible increased responsive-
ness to the muscle relaxant. Lithium - should not generally be given with
druretics. Diuretic agents reduce the renal clearance of lithium and add a high isk of lithium toxicity. Refer to the package insert for lithium preparations matory drugs - In some patients, the administration of a nonsteroidal anti-
inflammatory agent can reduce the diuretic, natriuretic. and antihypertensive effects of loop. potassium-sparing. and thiazide diuretics. Therefore. when
VASEREIIC and nonsteroidal ant-inflammatory agents are used concomi-
tantly, the patient should be observed closely to determine if the desired effect tantly. the patient should be observed closely to determine if the desired effect Pregnancy: Enalapril Maleate-Hydrochlorothiazide: Pregnancy Category $\mathrm{C}$
There was no teratogenicity in rats given up to $90 \mathrm{mg}$ kg day of enalapril (150
times the maximum human dose) in combination with $10 \mathrm{mg}, \mathrm{kg}$ day of
hydrochlorothiazide (2-1/2 times the maximum human dose) or in mice given p to $30 \mathrm{mg} / \mathrm{kg} /$ day of enalapril $(50$ times the maximum human dose) in com-
bination with $10 \mathrm{mg} / \mathrm{kg} / \mathrm{day}$ of hydrochlorothiazide $(2.12$ times the maximum human dose). At these doses. fetotoxicity expressed as a decrease in average
tetal weight occurred in both species. No fetotoxicity occurred at lower doses:
$30,10 \mathrm{mg} \mathrm{kg}$ day of enaiaprit-hydrochlorothiazide in rats and $1010 \mathrm{mg} / \mathrm{kg}$
There are no adequate and well-controlled studies in pregnant women.
VASERETIC (EEnalapril Maleate-Hydrochlorothiazide. MSO) should be used
during pregnancy only if the potential benefit justifies the potential risk to the Hydrochlorothiazide:

thrombocytopenia. and possibly other adverse reactions which have occurred

Nursing Mothers: ous reactions in nursing infants from hydrochiorothiazide. a decision should
be made whether to discontinue nursing or to discontinue VASERETIC, taking Pediatric Use: Satety and effectiveness in children have not been established ADVERSE REACTIONS

VASERETIC has been evaluated for safety in more
ing over 300 patients treated tor one year or more

In clinical trials with VASERETIC. no adverse experiences pecular to this combination drug have been observed. Adverse experiences that have occurred The most trequent cinical adverse experiences in controlled trials were: dizzi-
ness $(86 \%)$ headache $(5.5 \%)$, tatigue $(39 \%)$, and cough $(3.5 \%)$. Generally. ness $(8.6 \%)$, headache $(5.5 \%)$, tatigue $(39 \%)$, and cough $(3.5 \%)$. Genetally.
adverse experiences were mild and transient in nature. Adverse experiences occurring in greater than $2 \%$ of patients treated with
VASERETIC in controlled clinical trials are shown below

\begin{tabular}{|c|c|c|}
\hline & $\begin{array}{l}\text { Percest of Patients in C } \\
\text { VASERERIC } \\
\text { (n }=1.580 \text { ) } \\
\text { Incidence (discontinuation) }\end{array}$ & $\begin{array}{l}\text { tudies } \\
\text { Placebo } \\
\text { (n=230) } \\
\text { inodence }\end{array}$ \\
\hline $\begin{array}{l}\text { Dizziness } \\
\text { Headache } \\
\text { Fatigue } \\
\text { Cough } \\
\text { Muscle Cramps } \\
\text { Nausea } \\
\text { Asthenia } \\
\text { Orthostatic Effects } \\
\text { Impotence } \\
\text { Diarrhea }\end{array}$ & $\begin{array}{l}8.6(0.7) \\
5.5(0.4) \\
39(0.8) \\
3.5(0.4) \\
2.7(0.2) \\
2.5(0.4) \\
2.4(0.3) \\
2.3(<0.1) \\
2.2(0.5) \\
2.1(<0.1)\end{array}$ & $\begin{array}{l}4.3 \\
9.1 \\
2.6 \\
0.9 \\
0.9 \\
1.7 \\
0.9 \\
0.0 \\
0.5 \\
1.7\end{array}$ \\
\hline
\end{tabular}

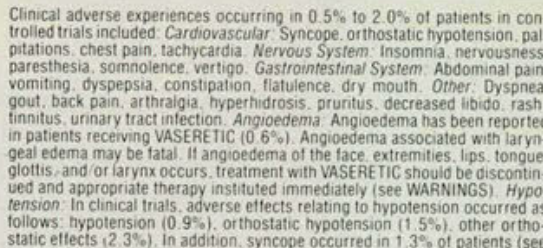
Clinical Laboratory Test Findings: Serum Electrolytes: See PRECAUTIONS in blood urea nitrogen and serum creatinine reversible upon discontinuation
of therapy were observed in about $0.6 \%$ of patients with essential hyperten-
sion treated with VASERETIC More marked increases have been reported sion treated with VASERETIC. More marked increases have been reported in other enalapril experience. Increases are more likely to occur in patients with
renal artery stenosis (see PRECAUTIONS), Serum Uric ACid, Glucose. Mag
nesium, and Calcium. See PRECAUTIONS. Hemoglobin and Hematocrit Small decreases in hemogiobin and hematocit (mean decreases of approxi-
mately $0.3 \mathrm{~g} \%$ and 1.0 vol $\%$, respectively) occur frequently in hypertensive
patients treated with VASERETIC but are rarely of clinical importance unless patients treated with VASERETIC bet are rarely of clinical importance unles.
another cause of anemia coexists. In cinical trials, less than $0.1 \%$ of patients
discontinued therapy due to anemia. Other (Causal Relationship Unoknown) Other adverse reactions that have been reported with the individual compo. nents are listed betow. Enalapni Maleate- Enalapril has been evaluated for
safety in more than 10.00 patients. In clinical Irials, adverse reactions which
occurred with enalapril were aiso seen with VASERETIC. However, since enala pril has been marketed. the following adverse reactions have been reportec
Renal: Renal dystunction, renal tailure. oliguria. Hematologic: Rare cases of neutropenia. thrombocytopenia, and bone marrow depression have been
reported in which a causal relationship to enalapril cannot be excluded
Hydrochlarothiazide-Body as a Whole: Weakness: Digestive: Anorexia. gas. Hydrochlorothiazide - Body as a Whole: Weakness: Digestive: Anorexia, gas.
tric irritation. cramping. jaundice (intrahtepatic cholestatic jaundice), pancre

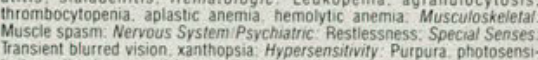
Iransient blurred vision. xanthopsia. Hypersensitivity : Purpura. photosensi.
tivity. urficaria, necrotizing angitis (vascultits and cutaneous vascuhtis).
lever, respiratory distress including pneumonitis and pulmonary edema, ana. HOW SUPPLIED

No. 3418 - Tablets VASERETIC 10-25 are red. squared capsule-shaped. com-
pressed tablets. coded MSO 720 on one side and VASERETIC on the other pressed tablets. coded MSO 720 on one side and VASERETIC on the othe:
Each tablet contains $10 \mathrm{mg}$ enalapril maleate and $25 \mathrm{mg}$ hydrochiorothiazide
They are supplied as follows: NDC 0006-0720-68 bottles of 100 (with desiccant)

MSD

MERCK Representative or see Prescribing informa

SHARP
DOHME Merck Sharp \& Dohme. Division of Merck \& C0. INC
J7VT04(302) 